TECHNICAL NOTE

\author{
Z.W. Zhang \\ L.J. Song \\ Q.F. Meng \\ Z.P. Li \\ B.N. Luo \\ Y.H. Yang \\ Z. Pei
}

\section{High-Resolution Diffusion-Weighted MR Imaging of the Human Lumbosacral Plexus and Its Branches Based on a Steady-State Free Precession Imaging Technique at 3T}

SUMMARY: 3D diffusion-weighted steady-state free precession imaging (3D DW-SSFP) with isotropic resolution was performed to delineate structures of the human lumbosacral plexus (LSP). 3D DWSSFP clearly revealed detailed anatomy of the LSP and its branches. Our data suggest that the sequence based on 3D DW-SSFP can be used for high-resolution MR imaging of the peripheral nervous system.
M $\mathrm{R}$ imaging evaluation of the normal peripheral nerve anatomy and diseases is mainly dependent on $2 \mathrm{D}$ MR imaging techniques, including T1-weighted spin-echo, T2weighted fast spin-echo, and inversion-recovery sequences with fat saturation. ${ }^{1-7}$ Although these techniques can produce excellent-quality images, they have limitations in describing deliberate orientations of the targeted nerves, in that section gaps in these techniques lead to lower anatomic coverage and less quantitative information. Furthermore, T2-weighted spin-echo techniques cannot image smaller nerves in the periphery because the nerves cannot be distinguished from blood vessels on T2-weighted spin-echo images. ${ }^{8} \mathrm{~A}$ combination of diffusion-weighted imaging (DWI) with fat-suppressed T2-weighted sequences has been proposed to overcome these technical limitations. Additionally, DWI based on spin-echo with an echo-planar readout has been used to evaluate the anatomy of the peripheral nervous system..$^{9-10}$ However, these conventional techniques have limited spatial resolution or low signal-to-noise ratio and often produce severe image distortion. ${ }^{8,11}$

It is difficult to reveal the detailed anatomy of the lumbosacral plexus (LSP) by using conventional MR imaging in that many important branches of the LSP are small, and contrast of the various anatomic structures is not sufficient to exhibit their discrete identification. The purpose of this study is to describe a high-spatial-resolution 3D diffusion-weighted steady-state free precession (3D DW-SSFP) sequence and prospectively evaluate its feasibility in human LSP imaging at 3T.

\section{Materials and Methods}

Twenty-four healthy subjects (10 men, 14 women; mean age, 42.2 years; range, 24-69 years) underwent high-spatial-resolution T2weighted 3D DW-SSFP imaging with a generalized autocalibrating partially parallel acquisition (GRAPPA) technique on a clinical 3T

Received July 23, 2007; accepted after revision August 24.

From the Departments of Radiology (Z.W.Z., Q.F.M., Z.P.L., B.N.L., Y.H.Y.) and Neurology (L.J.S., Z.P.), First Affiliated Hospital of Sun Yat-Sen University, Guangzhou, Guangdong, P.R. China.

Please address correspondence to Quanfei Meng, MD, Department of Radiology, 58\# Zhongshan 2nd Rd, Guangzhou, 510080, P.R. China; e-mail: cjr.mengquanfei@vip DOl 10.3174/ajnr.A0994
MR imager (Trio; Siemens Medical Solutions, Erlangen, Germany) with 2-element circularly polarized (CP) phased-array body coils combined with $\mathrm{CP}$ phased-array spinal coils.

After optimization of the sequence in 3 healthy subjects ( 1 woman and 2 men, 30-36 years of age), we used the following parameters: TR/TE, 14.16/4.61 ms; flip angle, $30^{\circ}$; FOV, $360 \mathrm{~mm}$; matrix, $512 \times$ 512; bandwidth, $184 \mathrm{~Hz}$ per pixel; fat suppression, water excitation technique; diffusion moments, $90 \mathrm{mT} / \mathrm{m}\left({ }^{*}\right) \mathrm{msec}$; 3D slab thickness, $256 \mathrm{~mm}$; voxel dimension, $0.8 \times 0.8 \times 0.8 \mathrm{~mm}$ (320 partitions); number of acquisitions, 1; acquisition time, 10 minutes 49 seconds. The diagram of the 3D DW-SSFP sequence used in this study is shown in Fig 1.

The postprocessing techniques, including maximum intensity projection and multiplanar reformation, were used to generate images of the LSP with the commercial software on the console of the MR imaging scanner. The datasets from 3D DW-SSFP sequences in all subjects were reconstructed in arbitrary planes oriented in relation to the course of the LSP and its branches. All images were analyzed by 2 experienced neuroradiologists. Each radiologist made initial evalu-

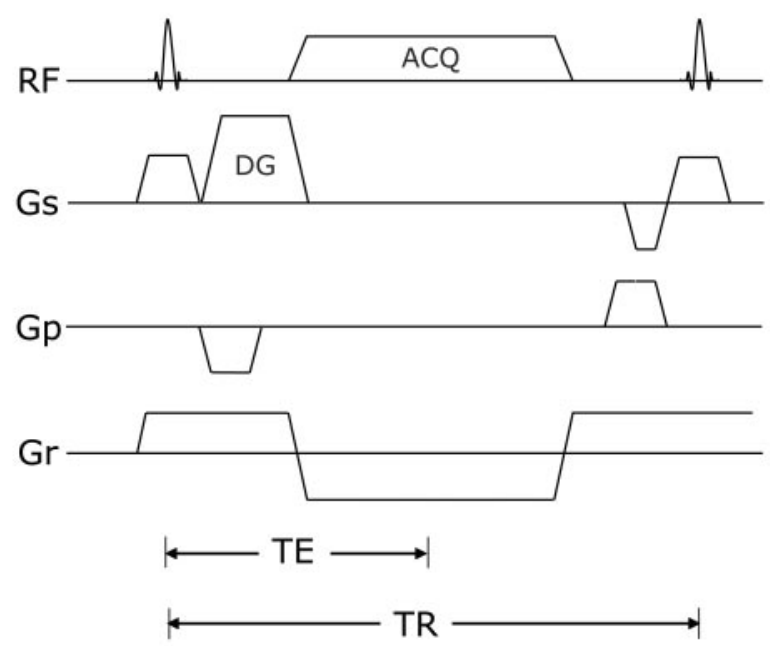

Fig 1. The 3D DW-SSFP sequence diagram used in the study. Diffusion gradients in the section direction were applied to reduce the signal intensity of fluid along the axis, and flow-compensation gradients in the read direction were applied to reduce motion sensitivity along this axis. DG indicates diffusion gradient; $A C Q$, acquisition; $R F$, radio-frequency; GS, section-selection gradient, Gp, phase-encoding gradient, Gr, read-out gradient. 

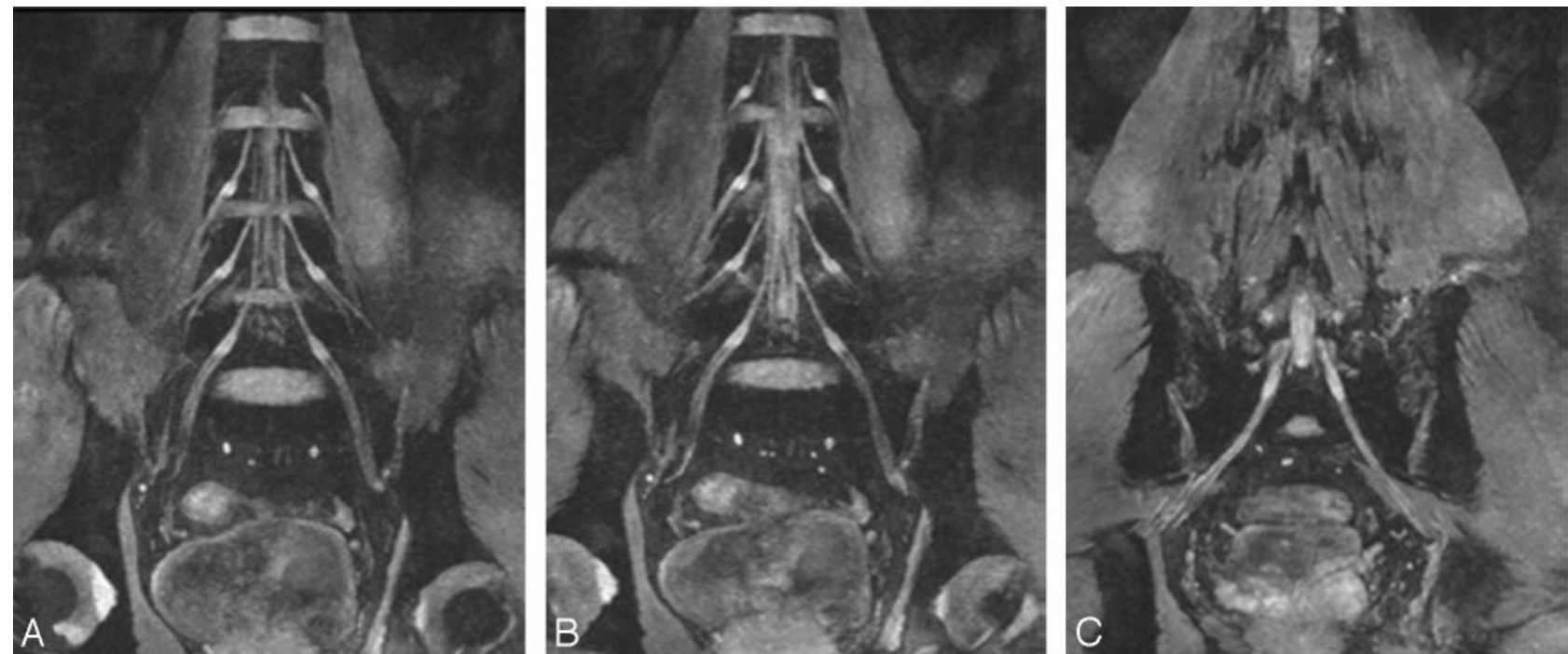

Fig 2. $A-C$, Thin-section $(20 \mathrm{~mm})$ coronal reconstruction MR images in a 45 -year-old volunteer obtained with a $3 D$ DW-SSFP sequence. These reconstruction planes clearly demonstrate the LSP and its components.
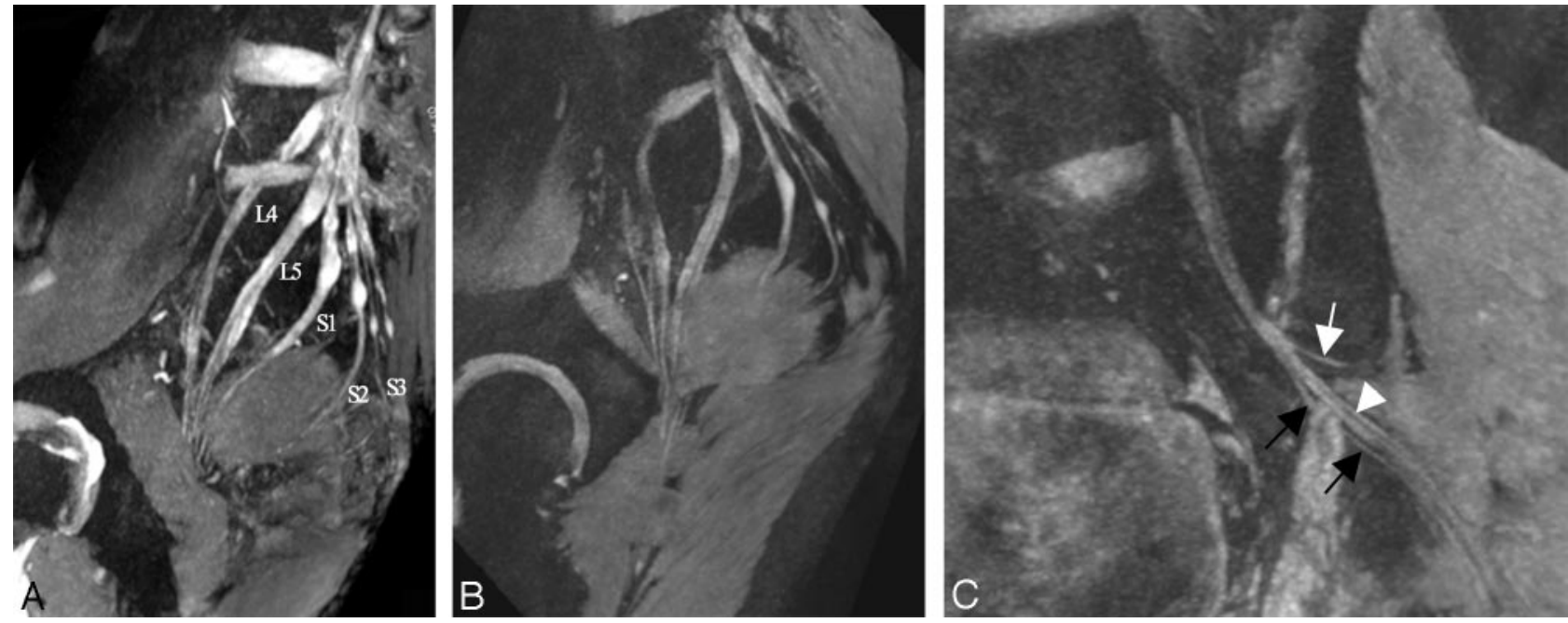

Fig 3. $A$, Thin-section $(20 \mathrm{~mm}$ ) oblique sagittal reconstruction plane in a 35 -year-old volunteer clearly demonstrates the components of the sacral plexus. $B$, Thin-section (20 mm) oblique sagittal reconstruction plane in a 28-year-old volunteer. The relationship between the nerves and the piriformis muscle is clearly defined. $C$, The tiny branches of LSP are shown. The white arrow indicates the superior gluteal nerve, the black arrow indicates the posterior cutaneous nerves of thigh, and the arrowhead indicates the sciatic nerve

ations independently, and any disagreements regarding final conclusions were resolved by consensus. The certainty of identifying the LSP and main branches was individually determined in each patient by using a 3-score scale system (2, identified with certainty; 1 , most probable identification; 0 , not identified).

The study protocol was approved by the local ethics committee and the review board of our department, and informed consent was obtained from all subjects before MR imaging examination.

\section{Results}

In arbitrary planes, the nerve roots from L1 to S4, the spinal nerve ganglion, and the proximal branches of the LSP were identified (score of 1 or 2 ) in 48 (100\%); the main branches of the lumbar plexus including the obturator nerves and the femoral and lateral femoral cutaneous nerves within the psoas major muscle were identified (score of 1 or 2) in 22 (92\%), 2 $(8 \%)$, and $16(67 \%)$, respectively. The main branches of the sacral plexus including the sciatic nerves, the posterior femoral cutaneous nerve, and the gluteal nerves were identified (score of 1 or 2$)$ in $24(100 \%), 20(83 \%)$, and $20(83 \%)$, respectively. Typical imaging results with the $3 \mathrm{D}$ DW-SSFP sequence are shown in Figs 2-4.

\section{Discussion}

The 3D DW-SSFP sequence in this study is a high-resolution diffusion-weighted T2-weighted method of steady-state gradient-echo imaging combined with fat-suppression and flowcompensation techniques. Our sequence has several advantages. First, the signal intensity of the sequence was formed by a spin-echo mechanism with characteristics of a spin-echo sequence and thus with dominating T2 contrast as compared with other spoiled or refocused gradient-echo techniques because the echo of the sequence is formed by a radio-frequency excitation that is unusual for gradient-echo sequences. Therefore, the influence of the local magnetic field in homogeneities can be reduced on the spin relaxation. ${ }^{12}$ 

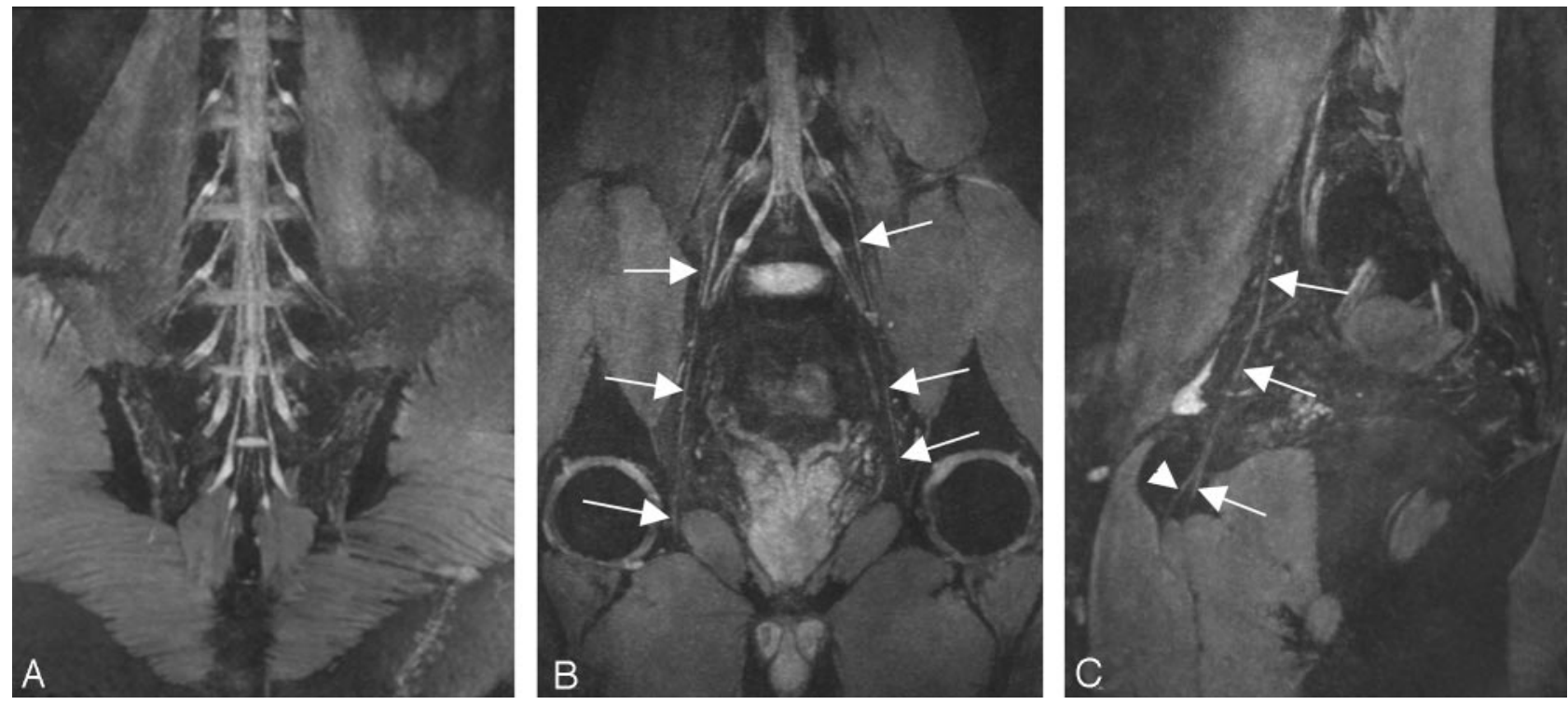

Fig 4. $A-C$, Curved thin-section $(20 \mathrm{~mm})$ coronal reconstruction MR images in a 29 -year-old volunteer obtained with a $3 D$ DW-SSFP sequence. The curved reconstruction plane $(A)$ along the ganglion clearly demonstrates the ganglions of LSP bilaterally, the curved reconstruction plane $(B)$ along the obturator nerve clearly demonstrates the obturator nerve (white arrow), and the curved reconstruction plane $(C)$ along the left obturator nerve clearly demonstrates the obturator nerve (white arrow) and its branches (white arrowhead).

Second, the detection of small nerve structures, especially those located near large vessels, is largely improved in that the sequence can completely eliminate the signal intensity from the vessels. The SSFP signal intensity heavily depends on the steady-state condition, and vessels do not produce signal intensity because it cannot construct the steady-state condition. ${ }^{13}$ In addition, the image contrast is further improved by using the DWI technique combined with the SSFP sequence, which completely suppresses the high signal intensity of the stationary fluid, such as urine and synovia. As a result, the signal intensity of stationary fluid is very low in contrast to the high signal intensity of nerves and the intermediate signal intensity of adjacent pelvic parenchymal structures on DWI images. Furthermore, the fat signal intensity is completely suppressed, and the chemical shift effect and motion-related phase errors are reduced by using a spectral spatial waterexcitation pulse. With this technique, only water is excited by using section-selective composite pulses, whereas lipid spins are left in equilibrium, thereby producing no signal intensity. ${ }^{14}$

Besides, this sequence is compatible with the GRAPPA technique at 3T. The combination of this sequence with GRAPPA allows performance of high-resolution imaging within a reasonable acquisition time, and image disturbances due to B0 field inhomogeneity in a high-field MR imager can be further decreased by the technique. Given that the thin contiguous sections and high isotropic resolution can depict tiny structures or lesions, any images can be reconstructed in arbitrary orientation by the multiplanar reconstructive technique without image degradation.

The limitations of this study include the motion, pulsation, and ghost artifacts in the lower abdomen. These artifacts can degrade or even disturb the quality of the images, especially the quality of images on the nerves close to the abdominal wall.

\section{Conclusion}

In the present study, 3D DW-SSFP clearly revealed detailed anatomy of the LSP and its branches. These data suggest that the sequence based on 3D DW-SSFP can be used for highresolution imaging of the peripheral nervous system.

\section{References}

1. Gierada DS, Erickson SJ, Haughton VM, et al. MR imaging of the sacral plexus: normal findings. AJR Am J Roentgenol 1993;160:1059-65

2. Blake LC, Robertson WD, Hayes CE. Sacral plexus: optimal imaging planes for MR assessment. Radiology 1996;199:767-72

3. Gierada DS, Erickson SJ. MR imaging of the sacral plexus: abnormal findings. AJR Am J Roentgenol 1993;160:1067-71

4. Maravilla K, Aagaard B, Kliot M. MR neurography: MR imaging of peripheral nerves. Magn Reson Imaging Clin N Am 1998;6:179-94

5. Filler AG, Howe FA, Hayes CE, et al. Magnetic resonance neurography. Lancet 1993;341:659-61

6. Maravilla KR, Bowen BC. Imaging of the peripheral nervous system: evaluation of peripheral neuropathy and plexopathy. AJNR Am J Neuroradiol 1998;19:1011-23

7. Moore KR, Tsuruda JS, Dailey AT. The value of MR neurography for evaluating extraspinal neuropathic leg pain: a pictorial essay. AJNR Am J Neuroradiol 2001;22:786-94

8. Filler AG, Maravilla KR, Tsuruda JS. MR neurography and muscle MR imaging for image diagnosis of disorders affecting the peripheral nerves and musculature. Neurol Clin 2004;22:643-82

9. Takahara T, Imai Y, Yamashita T, et al. Diffusion weighted whole body imaging with background body signal suppression (DWIBS): technical improvement using free breathing, STIR and high resolution 3D display. Radiat Med 2004;22:275-82

10. Tsuchiya K, Fujikawa A, Tateishi $\mathrm{H}$, et al. Visualization of cervical nerve roots and their distal nerve fibers by diffusion-weighted scanning using parallel imaging. Acta Radiol 2006;47:599-602

11. Colagrande S, Carbone SF, Carusi LM, et al. Magnetic resonance diffusionweighted imaging: extraneurological applications. Radiol Med 2006;111:392_ 419. Epub 2006 Apr 11

12. Hoffmann KT, Hosten N, Meyer BU, et al. CSF flow studies of intracrania cysts and cyst-like lesions achieved using reversed fast imaging with steadystate precession MR sequences. AJNR Am J Neuroradiol 2000;21:493-502

13. Taupitz M, Speidel A, Hamm B, et al. T2-weighted breath-hold MR imaging of the liver at 1.5 T: results with a three-dimensional steady-state free precession sequence in 87 patients. Radiology 1995;194:439-46

14. Schick F, Forster J, Machann J, et al. Highly selective water and fat imaging applying multislice sequences without sensitivity to B1 field inhomogeneities. Magn Reson Med 1997;38:269-74 